\title{
SANO95.2292C
}

\section{NEW FUNCTIONALIZED BLOCK COPOLYMERS FOR BONDING COPPER TO EPOXY}

\author{
Michael Kent, Randall Saunders, John Emerson, Michael Hurst \\ Sandia National Labs., Albuquerque, NM 87185 \\ RECEIVED \\ ABSTRACT \\ OCT 201995 \\ OSTI
}

We are exploring the use of functionalized block copolymers for bonding copper to epoxy in printed wiring boards. Our program involves four key elements: i) synthesis of suitable functionalized block copolymers; ii) characterization of the conformation of the copolymers at the relevant interfaces by neutron reflectivity; iii) spectroscopic measurements of chemical bonding, and iv) measurement of the mechanical properties of the interfaces. The copolymers are synthesized by living, ring-opening metathesis polymerization. This relatively new technique allows great flexibility for synthesis of functionalized block copolymers in that the initiators are relatively insensitive to a wide range of functional groups. Significant adhesion enhancement has been observed in lap shear tests.

KEY WORDS: Adhesives, Primers, Laminates

\section{INTRODUCTION}

Bonding copper to glass cloth reinforced FR4 epoxy is a critical step in the construction of multilayer printed wiring boards. Typically, the treated side of commercial $\mathrm{Cu}$ foils has large ( 10 micron) mushroom shaped nodules formed during electroplating. A zinc chromate coating is commonly used to provide a corrosion barrier and to enhance bonding with prepreg in the construction of the core laminates. The large surface roughness leads to mechanical interlocking with the epoxy and excellent adhesion. However, the nodular topography can lead to difficulties when the copper is etched, and also can limit line spacing and line widths. There is currently much interest in the industry for reducing or eliminating the nodular treatment. For multilayer PWBs, prepreg must be bonded to the untreated, smooth copper surfaces of the cores. This is usually accomplished with a hot alkaline sodium chlorite treatment to form a high surface area

This work was partially supported by the U.S. Department of Energy under contract DEAC04-94AL85000.

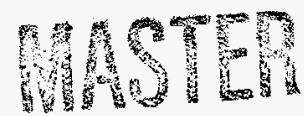




\section{DISCLAIMER}

Portions of this document may be illegible in electronic image products. Images are produced from the best available original document. 
dendritic cupric oxide layer. While this treatment is quite successful in promoting adhesion, it is costly, time consuming, and involves harsh chemicals. The goal of the present work is to increase the chemical component of adhesion at the copper/epoxy interface in order to i) reduce the profile height required for the nodular surfaces and ii) enhance or even replace the brown oxide treatment of the smooth $\mathrm{Cu}$ surfaces.

To this end we are exploring the use of functionalized diblock copolymers as adhesion promoters. We envision several potential advantages of functionalized block copolymers over small molecule coupling agents. Greater control over the structure of the interphase region should result through careful design of the backbone of the copolymer. The number of chains per area, the degree of entanglement between the copolymer and the polymer matrix, the number of sites per chain able to attach to the substrate, and the hydrophobicity of the interphase region can all be strongly affected by the choice of block lengths and the monomer sequence. In addition, entanglement between the copolymer and the polymer matrix, if achieved, should contribute significantly to adhesive strength.

Below we describe the synthesis of the block copolymers by living, ring-opening metathesis polymerization (ROMP), characterization data obtained by neutron reflectivity and FTIR, and results of adhesion tests.

\section{EXPERIMENTAL}

\section{Synthesis}

ROMP has been known for many years. However, the synthesis of block copolymers by ROMP became possible only recently when Grubbs [1] and Schrock [2] developed molybdenum and titanium based initiators capable of living polymerization. The molybdenum based initiator is capable of tolerating a wide variety of chemical functionalities on norbornene-type monomers, and thus lends itself to the synthesis of well-defined, functionalized block copolymers that can be tailored to a wide variety of interfaces.

All reactants were purchased from Aldrich Chemical Company. Tetrahydrofuran was used as the polymerization solvent, and was vacuum distilled from a sodium/benzophenone solution immediately prior to use. The molybdenum based initiator ((2,6-diisopropylimido)neophylidine-molybdenum-bis-t-butoxide) was purchased from Strem and used without further purification. All polymerizations were performed under an inert atmosphere in a Vacuum Atmospheres dry box.

The synthesis of the two monomers, (5-(n-methylimidazole)-2-norbornene and 5-(tbutylaminomethyl)-2-norbornene, will be described in detail elsewhere. Both were dried by distilling from and storing over calcium hydride or sodium hydride, and filtering through activated neutral alumina immediately prior to use. 
A partially deuterated form of the imidazole monomer, used in the neutron reflection experiments, was prepared following the same reaction scheme as that for the protonated imidazole monomer, but starting with a partially deuterated dicyclopentadiene precursor. This sample contained 4 to 5 deuterium atoms per repeat unit.

The block copolymer synthesized for this study is shown below.

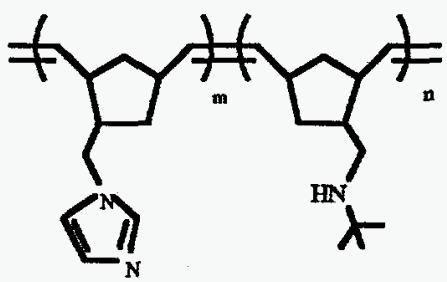

Typically a total of $1 \mathrm{~g}$ of copolymer was formed in about $15 \mathrm{mls}$. dry THF. An appropriate amount of the first monomer was initially stirred with the molybdenum initiator. Upon complete consumption, the second monomer was then added. After the polymerization of the final block, the living chain ends were terminated by the addition of benzaldehyde. The copolymers were characterized by proton $\mathrm{nmr}$, which showed complete consumption of the amine functionalized monomer, but only partial consumption of the imidazole monomer. The latter was the last block in the polymerization sequence, and thus did not destroy the continuity of the amine block. The block copolymers were precipitated from acetonitrile, dried, and redissolved in chloroform for future use.

For the imidazole functionalized blocks, the actual molecular weights $(10 \mathrm{~kg} / \mathrm{mol})$ were slightly lower than the targeted values $(15 \mathrm{~kg} / \mathrm{mol})$. The amine functionalized blocks are assumed to have the targeted molecular weight based upon the observation of total monomer consumption.

\section{Conformation of adsorbed copolymers by neutron reflectivity}

Since both the imidazole and amine functionalities have an affinity for the copper surface, it is essential to determine whether the blocks order into layers upon adsorption to a copper substrate, with one block adsorbing preferentially, or whether they adsorb in a disordered conformation with both blocks adsorbing at the surface. To address this question, we prepared copolymer samples with partially deuterated imidazole blocks and examined adsorbed films of these copolymers by neutron reflectivity. With this technique the neutron refractive index profile normal to the surface is obtained with $\sim 5$ $\AA$ resolution. The selective deuteration allows one to distinguish between the two blocks. The copolymers were adsorbed from solution onto silicon wafers which had been previously sputter-coated with smooth, thin $(\sim 250 \AA)$ copper films. Following the adsorption step, the samples were thoroughly washed with $\mathrm{MeOH}$ and THF to remove any copolymer not strongly adsorbed to the surface. 
Results for the diblock copolymer with targeted block lengths $15 \mathrm{~K}-15 \mathrm{~K}$ adsorbed from $0.002 \mathrm{~g} / \mathrm{ml}$ solution in $\mathrm{MeOH}$ are shown in Figure 1. The main effect of the adsorbed copolymer on the reflectivity is to shift the fringes to lower $\mathrm{q}$. This is consistent with the imidazole block selectively adsorbing to the surface. The result for the diblock copolymer with targeted block lengths $15 \mathrm{~K}-40 \mathrm{~K}$ adsorbed from $0.002 \mathrm{~g} / \mathrm{ml}$ solution in $\mathrm{MeOH}$ is also shown in Figure 1. The curve falls on top of that for the sample with the $15 \mathrm{~K}-15 \mathrm{~K}$ copolymer. This is expected only if the blocks form separate layers with the imidazole block adsorbed to the surface. Since the amine blocks are not deuterated they contribute little to the reflectivity in this configuration, and so copolymers with differing amine block lengths yield nearly the same reflectivity curve. Thus, these data suggest that the copolymer adsorbs in a monolayer film, and that the blocks form separate layers with the imidazole block adsorbed to the surface and the amine block free to bond with the epoxy.

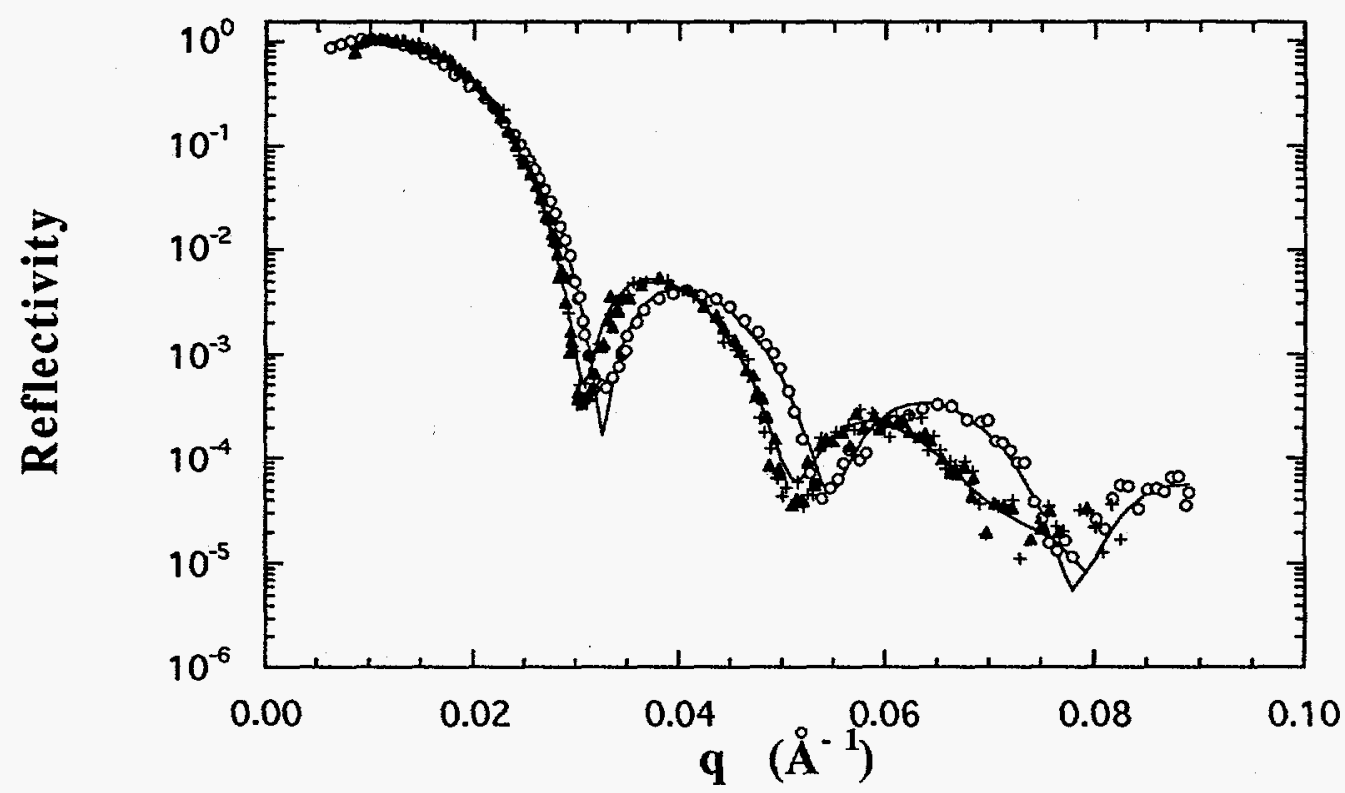

Figure 1. Neutron reflectivity for bare $\mathrm{Cu}$ film - (open circles), $\mathrm{Cu}$ film with 15-15 copolymer adsorbed - (solid triangles), and $\mathrm{Cu}$ film with $15-45$ copolymer adsorbed $-(+)$

\section{Reactivity of the amine functionalized monomer with FR4 epoxy by FTIR}

We have examined the reactivity of the amine functionalized monomer toward FR4 epoxy with FTIR. A peak at $920 \mathrm{~cm}^{-1}$ is characteristic of the epoxide ring vibration and is used to detect the extent of the crosslinking reaction. In Figure $2 \mathrm{a}$ we show the spectra for the uncured FR4 epoxy resin. For this sample, all components of the FR4 resin were present (Shell EPON 1124-A-80, dicyandiamide, 2-methyl imidazole) but the sample was not yet heated. We observe a strong peak at $920 \mathrm{~cm}^{-1}$, which indicates that very little reaction of the epoxide groups with DICY has occurred. In Figure $2 b$, 
we show the spectra for the fully cured FR4 epoxy resin. The sample is identical to that in Fig. 1a except that the sample has been heated to $177^{\circ} \mathrm{C}$ for 2 hours. The peak at $920 \mathrm{~cm}^{-1}$ is now absent, which indicates that nearly all the epoxide groups have reacted. In Figure 2c we show the spectra for a sample identical to that in Figure $2 b$, except that DICY has been replaced by the amine functionalized monomer. We observe in Fig $2 \mathrm{c}$ that the epoxide peak at $920 \mathrm{~cm}^{-1}$ has almost completely disappeared, indicating that the amine functionalized monomer is indeed highly reactive toward the epoxy resin.
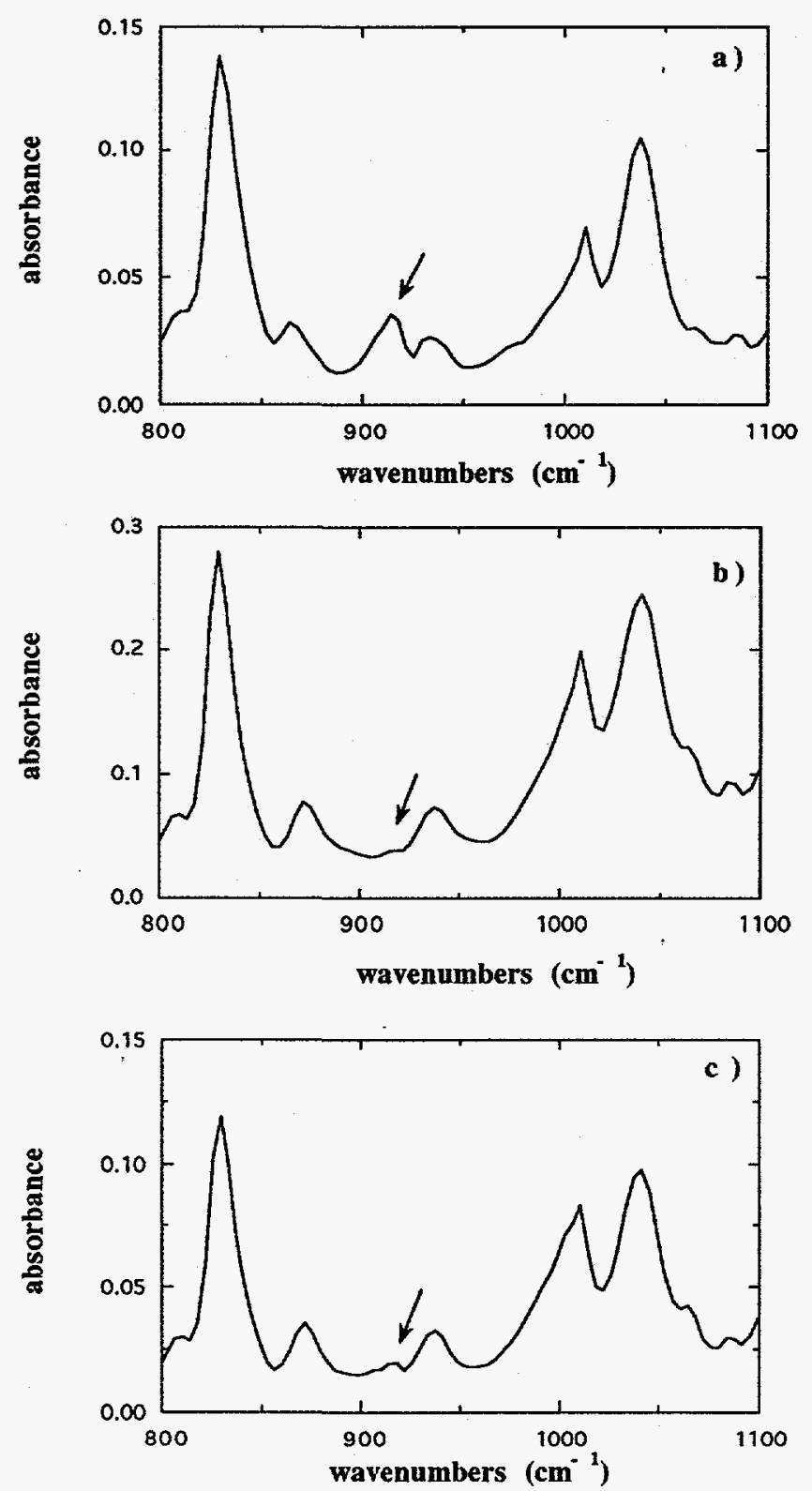

Figure 2. FTIR spectra for a) uncured FR4 epoxy, b) cured FR4 epoxy, and c) cured FR4 with DICY replaced by the amine functionalized monomer 


\section{Adhesion testing}

Lap shear coupons were machined from copper blocks. The bonded surfaces of all samples were milled to a $0.8 \mu \mathrm{m}$ average roughness finish, microetched using Shipley's PREPOSIT ETCH 748 solution, and then rinsed exhaustively with deionized water. A particular point of interest in this initial study is whether adhesion enhancement is greatest when the copolymer is adsorbed onto bare $\mathrm{Cu}$ or onto the native oxide. Xue, et al., ${ }^{3}$ have examined the growth of the native oxide on copper after acid etching and rinsing by surface enhanced Raman spectroscopy. They showed that the native oxide on copper becomes observable after $\sim 30$ minutes, and reaches a limiting thickness after $\sim 2$ hours. Therefore, after the deionized water rinse, sample set \#1 was immediately placed into a $0.002 \mathrm{~g} / \mathrm{ml}$ methanol solution of the block copolymer in order to test the adhesion to bare copper. Sample set \#2 was left in air for 3 hours after rinsing, and then placed into the methanol solution of the block copolymer. Both sets of samples were left in the block copolymer solution overnight, then washed exhaustively with methanol, and rollcoated with FR4. Sample set \#3 was prepared with the block copolymer dissolved into the FR4 resin at $1.8 \mathrm{wt} \%$, replacing the accelerator, 2 methyl imidazole. The final two sets of samples were controls. One set of control samples was composed of FR4 rollcoated directly onto the native oxide of coupons which had been microetched and rinsed. The second set of control samples was composed of coupons which were microetched, rinsed, and then treated to give the industry standard brown oxide. After curing ( 10 mins. at $60^{\circ} \mathrm{C}, 2 \mathrm{hrs}$. at $177^{\circ} \mathrm{C}$ ), the samples were cooled to room temperature and pulled with an Instron. All sample sets were composed of 5 coupons. The data are displayed in Figure 3 . We observe that i) the force to break samples \#2 and \#3 was significantly higher than that for the brown oxide treated samples, and ii) the adhesion promotion was greater when the copolymer was bonded to the native oxide rather than to bare copper. Further testing involving thermal shocking is currently underway.

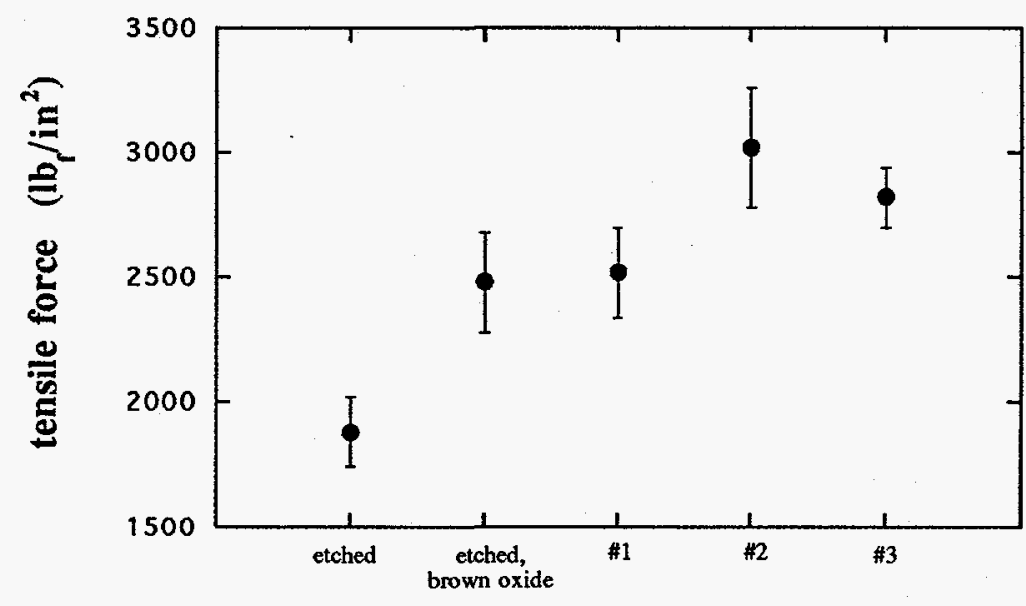

treatment

Figure 3. Results of lap sheer test 


\section{REFERENCES}

1. R.H. Grubbs and W. Tumas, Science, 243, 902, (1989).

2. R. R. Schrock, Acc. Chem. Res., 23, 158, (1990).

3. G. Xue, Y. Lu, and G. Shi POLYMER, 35 (12), 2488, (1994).

\section{ACKNOWLEDGMENTS}

This work benefited from the use of the POSY II reflectometer at the Intense Pulsed neutron Source at Argonne National Labs, and was supported by the U.S. D.O.E. under contracts DE-AC04-94AL85000 and W-31-109-ENG-38.

\section{DISCLAIMER}

This report was prepared as an account of work sponsored by an agency of the United States Government. Neither the United States Government nor any agency thereof, nor any of their employees, makes any warranty, express or implied, or assumes any legal liability or responsibility for the accuracy, completeness, or usefulness of any information, apparatus, product, or process disclosed, or represents that its use would not infringe privately owned rights. Reference herein to any specific commercial product, process, or service by trade name, trademark, manufacturer, or otherwise does not necessarily constitute or imply its endorsement, recommendation, or favoring by the United States Government or any agency thereof. The views and opinions of authors expressed herein do not necessarily state or reflect those of the United States Government or any agency thereof. 